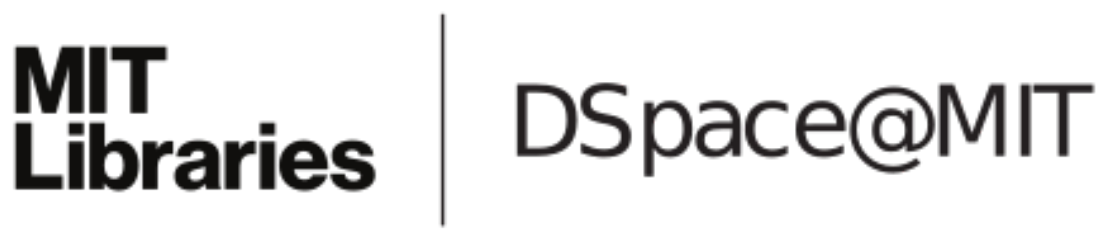

\author{
MIT Open Access Articles
}

Validation and application of an ensemble Kalman filter in the Selat Pauh of Singapore

The MIT Faculty has made this article openly available. Please share how this access benefits you. Your story matters.

Citation: Wei, Jun, and Paola Malanotte-Rizzoli. "Validation and Application of an Ensemble Kalman Filter in the Selat Pauh of Singapore." Ocean Dynamics 60.2 (2010) : 395-401-401. Copyright (c) 2009, Springer-Verlag

As Published: http://dx.doi.org/10.1007/s10236-009-0253-y

Publisher: Springer

Persistent URL: http://hdl.handle.net/1721.1/64679

Version: Author's final manuscript: final author's manuscript post peer review, without publisher's formatting or copy editing

Terms of use: Creative Commons Attribution-Noncommercial-Share Alike 3.0 


\title{
Validation and application of an ensemble Kalman filter in the Selat Pauh of Singapore
}

\author{
Jun Wei • Paola Malanotte-Rizzoli
}

Received: 20 July 2009 / Accepted: 24 November 2009

(C) Springer-Verlag 2009

\begin{abstract}
The effectiveness of an ensemble Kalman filter (EnKF) is assessed in the Selat Pauh of Singapore using observing system simulation experiment. Perfect model experiments are first considered. The perfect model experiments examine the EnKF in reducing the initial perturbations with no further errors than those in the initial conditions. Current velocity at 15 observational sites from the true ocean is assimilated every hour into the false ocean. While EnKF reduces the initial velocity error during the first few hours, it fails after one tidal cycle (approximately $12 \mathrm{~h}$ ) due to the rapid convergence of the ensemble members. Successively, errors are introduced in the surface wind forcing. A random perturbation $\varepsilon$ is applied independently to each ensemble member to maintain the ensemble spread. The assimilation results showed that the success of EnKF depends critically on the presence of $\varepsilon$, yet it is not sensitive to the magnitude of $\varepsilon$, at least in the range of weak to moderate perturbations. Although all experiments were made with EnKF only, the results could be applicable in general to all other ensemble-based data assimilation methods.
\end{abstract}

Keywords Ocean modeling · Data assimilation ·

Ensemble Kalman filter

Responsible Editor: Leo Oey

J. Wei $(\triangle) \cdot$ P. Malanotte-Rizzoli

Department of Earth, Atmospheric and Planetary Sciences,

Massachusetts Institute of Technology,

Cambridge, MA 02139, USA

e-mail: junwei@mit.edu

P. Malanotte-Rizzoli

e-mail: rizzoli@mit.edu

\section{Introduction}

Numerical ocean models have become capable of simulating the four dimensional evolution of the ocean with the flexible resolution that is essential to reproduce and understand the processes not captured by the limited observations. In spite of this progress, ocean models still contain errors because of the incomplete representation of ocean physics, numerical implementation, insufficient resolution, as well as errors in forcing functions and observations. Thus, it is necessary to correct for them, and this can be done through data assimilation. Data assimilation methods synthetize the model solution with the available limited observations to obtain an optimal solution (analysis) which can be used as the new initial condition for model forecasting. In general, the analysis procedure minimizes the misfit between the model states and the observations using least-square methods. In this study, we focus on the ensemble Kalman filter (EnKF), one of the most advanced sequential assimilation methods, introduced in oceanography by Evensen (1994).

The original Kalman filter is developed for a linear system, and the extended Kalman filter (EKF) extends its basic algorithm to nonlinear problems by linearizing the nonlinear function around the current estimate. For a realistic ocean model, however, it is difficult to directly apply the EKF because ocean dynamics is strongly nonlinear and any linearization fails very quickly. Thus, a number of suboptimal approximations have been proposed. Among them are the filters based on ensemble estimation (Evensen 1994; Houtekamer and Mitchell 1998; Tippett et al. 2003; Zang and Malanotte-Rizzoli 2003; Chen et al. 2009).

The EnKF predicts a flow-dependent error covariance through a set of ensemble forecasts computed directly from 
nonlinear models. We do not include a detailed illustration of the EnKF procedure as it has been amply discussed in the published literature and can be found in the references quoted above. The success of EnKFs for large scales is in part due to the global and continuous coverage of satellite data which provides stringent constraints on the ocean models. In contrary, the studies of Kalman filters in coastal areas are very limited. Chen et al. (2009) present an extensive application of the ensemble filters with limited, point-wise measurements in three coastal problems. They used the twin experiment approach considering a perfect model and 20 ensemble members. They showed that the ensemble-based filters (traditional EnKF and ensemble square root Kalman filter) are very successful in an idealized tide- and buoyancy-driven problem in an estuary. In this study, we examine the EnKF scheme in a realistic coastal region, the Selat Pauh of Singapore. Perfect and perturbed model experiments are carried out to examine the reliability and limitation of the EnKF. The paper is organized as follows. Section 2 describes the study area and the ocean model. Section 3 briefly discusses the experimental design. Section 4 presents the observing system simulation experiment (OSSE) results, and Section 5 gives a conclusive discussion.

\section{Study area and ocean model}

In Malay, "selat" means strait or channel. Selat Pauh is located to the west of the Singapore Strait and is surrounded by four major islands, Pulau Bukom, Pulau Semakau, Pulau Sudong, and Pulau Busing (Fig. 1). Selat Pauh is about $8 \times 2 \mathrm{~km}$ in the west-east and south-north directions, respectively. The maximum depth in the channel is about $25 \mathrm{~m}$. The circulation in the Selat Pauh is dominated by barotropic tidal flow with a weak vertical stratification. Its subtidal circulation is characterized by vortices induced by tide-island interactions and the wind (monsoon) driven circulation.

The model used in this study is an unstructured grid, three-dimensional, free surface, primitive-equation finite volume coastal ocean model, developed by Chen et al. $(2003,2006 a, b)$ to which the reader is referred for details. The model grid covers the large Selat Pauh area and is closed by a circular open boundary (Fig. 1). Horizontal resolution is $20 \mathrm{~m}$ along the coast of the islands and increases to $500 \mathrm{~m}$ at the open boundary. The model is configured with five sigma levels in vertical. We note that even though the configuration of five layers is not enough for a 3-D baroclinic simulation, it is justifiable in our case as only barotropic velocities are considered, and it is efficient for testing the EnKF. The model simulation is driven by the realistic tide along the open boundary and the

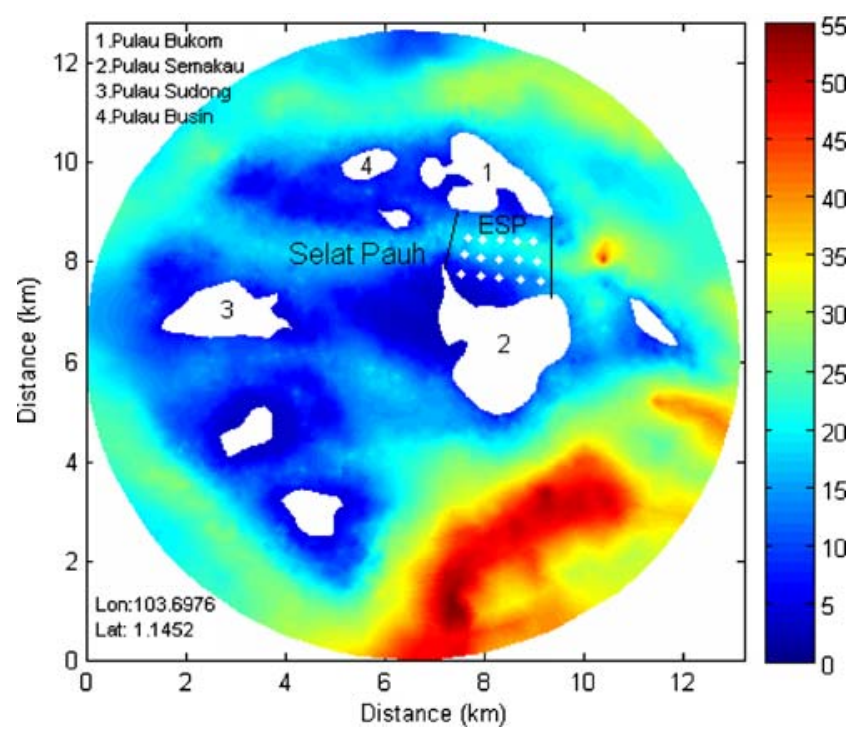

Fig. 1 Large Selat Pauh model domain and bottom topography. Fifteen observational sites are marked in the east Selat Pauh (ESP) sub-domain. The latitude and longitude of origin $(0,0)$ is marked

wind stress at the surface. The tidal forcing is obtained from a regional Singapore Strait model (courtesy of the Tropical Marine Science Institute of the National University of Singapore), and the wind stress is from the 6-h National Centers for Environmental Prediction reanalysis, uniform over the domain. The model is spun up under homogeneous condition and is integrated from January 3 to 12, 2007, period for which we have the boundary tidal forcing. Therefore, the model is used in its barotropic version, and the assimilated data are the barotropic velocities.

We chose east Selat Pauh (hereafter referred as ESP domain which is marked in Fig. 1) as assimilation domain to focus on small scales. The control run (true ocean) and the simulation with wrong initial conditions and, successively, wrong wind forcing (false ocean) are integrated with the large Selat Pauh domain (including the ESP domain), while the analysis procedure (assimilation) is conducted only in the ESP domain. The barotropic velocities are observed from the true ocean and assimilated into the false ocean at 15 observational sites in the ESP domain (Fig. 1).

\section{Experimental design}

The algorithm of EnKF is described in Kalnay (2003) to which the reader is referred for details. The EnKF theory is based on the statistical estimation of sufficient ensemble members. Large ensemble size however requires large computational resource. In this study, we use 20 ensemble members following Chen et al. (2009), which showed its efficiency in nonlinear tidal and buoyancy-driven problems. 
A localization scheme is necessary for coastal ocean data assimilations. First, from the statistical point of view, the forecast error covariance contains large sampling errors amongst those distant grid points (Anderson 2001; Hamill et al. 2001), and, theoretically, the localization scale should be consistent with the dominant scale of the flow structure. We use a localization scheme introduced by Gaspari and Cohn (1999). A smooth function with $500 \mathrm{~m}$ cut-off radius is applied to each observation point when calculating the error covariance. The cut-off radius defines the utmost distance that an observation might affect the neighboring grid points.

OSSE with twin experiment approach provides a complete knowledge of the true ocean state and is widely used to assess the data assimilation method. All OSSE experiments in this study are listed in Table 1 . The perfect model experiments examine the EnKF in reducing the initial perturbations with no other errors. There are three model runs: the true ocean, the false ocean, and the assimilation. The true ocean is the unconstrained model simulation. The false ocean run is integrated in the same period but starting from different, and wrong, initial conditions uncorrelated with the initial condition of the true ocean. In the assimilation run, barotropic velocity from the true ocean is assimilated every hour into the false ocean. The perturbed model experiments consider errors both in the initial conditions and in the surface wind forcing. The true ocean is the same as the perfect model. In the false ocean, the wind stress is perturbed, and the perturbed wind is constructed from the true wind plus a weighted difference between the true wind and the wrong wind

$W_{i}=W_{\text {true }}+\left(W_{\text {wrong }}-W_{\text {true }}\right) \times\left(\alpha+\varepsilon_{i}\right) \quad i=1,2 \ldots$ ens

$W_{\text {true }}$ is the wind actually observed in the period January 3-12, 2007 and $W_{\text {wrong }}$ is the wind observed during the successive 10 days, January 12-21, 2007. Two perturbation factors ( $\alpha$ and $\varepsilon$ ) are used to control the wind perturbations; $\alpha$ controls the major difference between the true wind and the perturbed wind, which is the same for all ensemble members, and $\varepsilon_{s}$ controls the magnitude of the added random perturbation which is different for each ensemble member. Note that in real applications, only $\varepsilon$ is needed because the wind error (equivalent to $\alpha$ here) is usually unknown. We consider errors only in the wind forcing and not in the open boundary conditions for the following reason. Errors in open boundary forcing data become important in areas not tidally driven, where mesoscale eddies can induce random fluctuations difficult to measure. The Selat Paul channel is fully tidally driven, and the tide data are excellent as a 23-year tidal record exists at the Tanjong Pajar gauge of Singapore. The tide has been very faithfully reconstructed all around the islands of Singapore through harmonic analysis (Tkalich et al. 2009). On the other side, the wind data are very poor as no wind observations are taken in the channels of the island with important daily excursion.

\section{OSSE results}

The true ocean state is the model simulation of January 8 12,2007 . The velocity field is mainly controlled by the boundary tidal forcing and slightly modified by the wind forcing. The false ocean run is initialized with a set of wrong initial conditions (ensemble members) uncorrelated with the initial condition of the true ocean. The initial ensemble members of the false ocean are constructed from a random sampling of the model states in the period January 3-7. Each ensemble member is integrated forward without data assimilation, and the mean of ensemble forecasts is regarded as the false ocean state and is used to calculate the root mean square (RMS) errors with respect to the true ocean. Figure 2a,b shows the RMS errors of the false ocean (only first 48-h results are showed). The initial velocity error decreases dramatically in the first $12 \mathrm{~h}$ (approximately one tidal cycle) and remains at the level of

Table 1 Experiment list

\begin{tabular}{lll}
\hline Perfect model experiments & True ocean & Unconstrained model simulation \\
& False ocean & Forecast with wrong initial conditions \\
& Assimilation & Assimilation with velocity from true ocean \\
Perturbed model experiments & False ocean & Forecast with perturbed wind stress \\
& Experiments of $\varepsilon$ & Case $1: \alpha=0.5, \varepsilon_{s}=0.5$ \\
& Case 2: $\alpha=0.5, \varepsilon_{s}=0.2$ \\
& Experiments of $\alpha$ & Case 3: $\alpha=0.5, \varepsilon_{s}=0$ \\
& Case 4: $\alpha=0.5, \varepsilon_{s}=0.2$ \\
& Case 5: $\alpha=0.2, \varepsilon_{s}=0.2$ \\
& Case 6: $\alpha=0, \varepsilon_{s}=0.2$ \\
\hline
\end{tabular}


Fig. 2 Perfect model experiments. a Root mean square (RMS) errors at observational sites, (b) RMS errors over the east Selat Pauh domain, (c) normalized error, and (d) ensemble spread

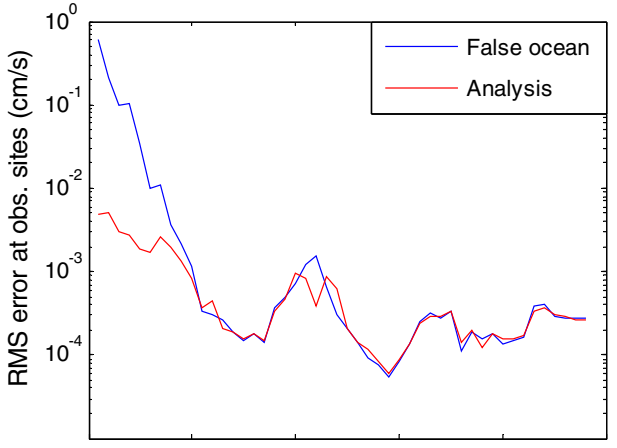

(a)

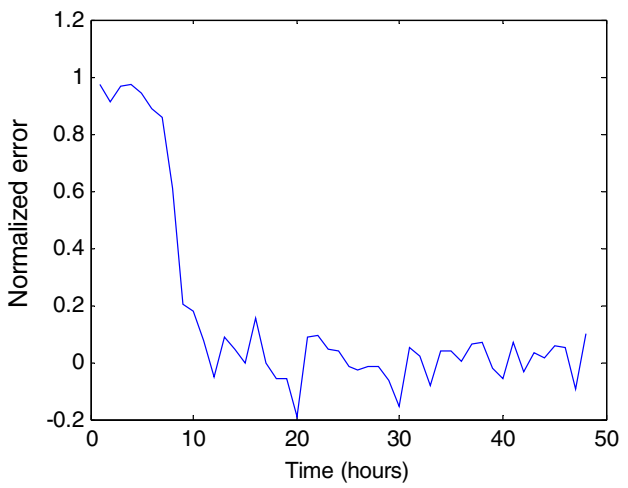

(c)

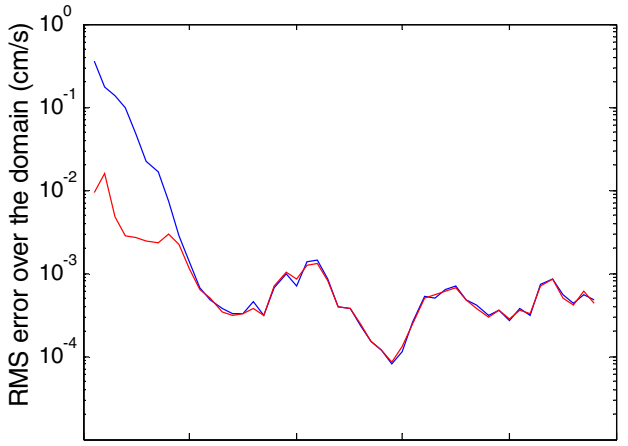

(b)

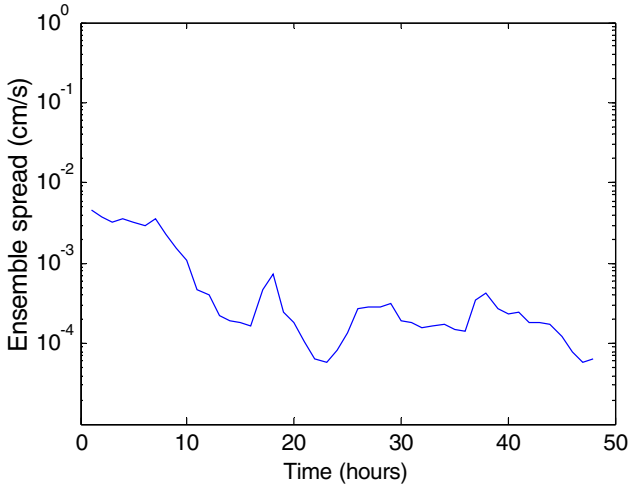

(d)
$10^{-3} \mathrm{~cm} / \mathrm{s}$. Since the model is the same for the true and false ocean simulations, the false ocean has only the initial errors, and their rapid dissipation is due to the enforcement of the "true" boundary tidal forcing.

In the assimilation experiment, the observations extracted from the true ocean include an observational error for all the ensemble members. Random noise is in fact added to the data, specified as $1 \%$ of the standard deviation of the true solution relative to the mean. The random noise is derived from a Gaussian distribution with zero mean and unit standard deviation. Figure 2a,b also show the analysis RMS errors after data assimilation. The analysis RMS errors are smaller than the false ocean errors in the first $12 \mathrm{~h}$ due to the data assimilation, while afterward, they quickly converge to the false ocean errors implying the subsequent ineffectiveness of the EnKF. We also calculate the normalized error $\left(E_{n}\right)$ to reveal the error reduction due to the assimilation only as

$E_{n}=\left[1-\frac{\mathrm{RMS}(\text { analysis })}{\mathrm{RMS}(\text { false_ocean })}\right]$

which is shown in Fig. 2c. The normalized error is above 0.8 in the first few hours and gradually decreases to around zero, indicating that indeed the EnKF fails to reduce the errors with respect to the false ocean after $12 \mathrm{~h}$. The ineffectiveness of the EnKF is due to the rapid convergence of the ensemble members indicated by the decrease of ensemble spread. The ensemble spread is calculated as

$E_{\text {spread }}=\sqrt{\frac{1}{N} \sum_{i=1}^{N} \frac{1}{M} \sum_{j=1}^{M}(x-\bar{x})^{2}}$

where $x$ is the state variable, $M$ is ensemble size, and $N$ is the total number of model grid points for state variables. Figure $2 \mathrm{~d}$ shows the ensemble spread over the ESP domain. The spread decreases from $10^{-2} \mathrm{~cm} / \mathrm{s}$ at $t=0$ to $10^{-4} \mathrm{~cm} / \mathrm{s}$ at hour 12 and remains at the same level afterward. This result is not surprising. We point out that in the assimilation experiment, there are only velocity errors in the initial condition, $\mathrm{O}(1 \mathrm{~cm} / \mathrm{s})$. They decrease rapidly to $0.01-$ $0.001 \mathrm{~cm} / \mathrm{s}$ as the model solution quickly converges to the true ocean because the tidal boundary forcing and wind forcing are correct. Even though after each analysis procedure the analysis is again randomly perturbed to a set of new ensemble members used as initial conditions for the next forecast, the initial perturbations are mostly swamped out by the dominant tidal forcing during the next 1-h model forecasting.

In the perturbed model experiments, the wind forcing is modified according to Eq. 1. Figure $3 \mathrm{a}$ shows the difference (square root of $\left[\left(U_{\text {wrong }}-U_{\text {true }}\right)^{2}+\left(V_{\text {wrong }}-V_{\text {true }}\right)^{2}\right]$ ) between the true wind and the wrong wind. The maximum 
Fig. 3 Perturbed model experiments. a Difference between the wrong wind and the true wind, (b) root mean square errors over the east Selat Pauh domain, (c) normalized error, and (d) ensemble spreads
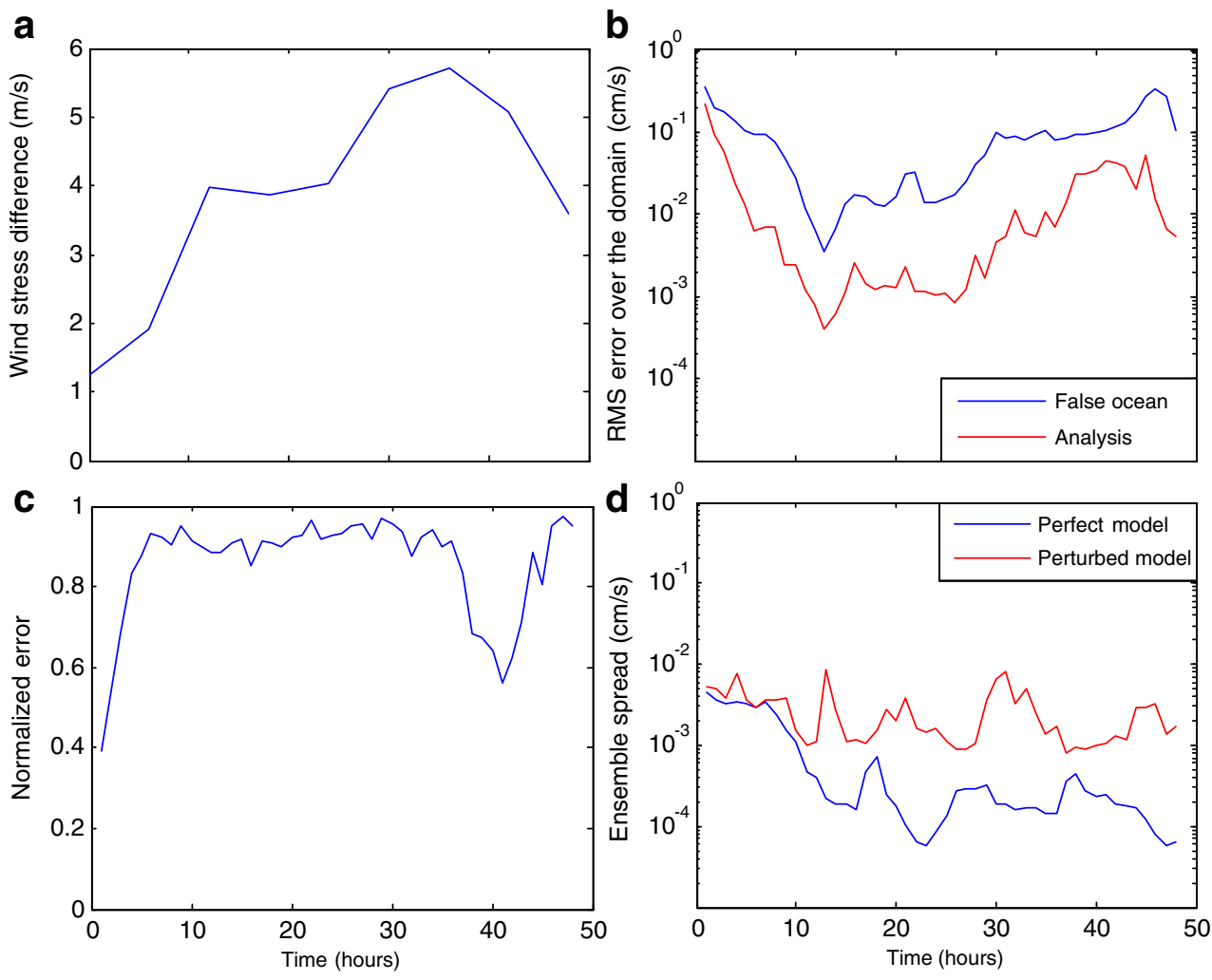

difference is about $6 \mathrm{~m} / \mathrm{s}$ at hour 36. In Eq. 1, $\varepsilon$ is a random number with zero mean and standard deviation of $\varepsilon_{s}$ for which different values are used as shown in Table 1. The false ocean is calculated as the mean of the ensemble members without data assimilation. Different from the perfect model case, the perturbed model experiments contain an imperfect model (with wind forcing errors) and inconsistent errors (consequence of both initial and wind stress errors). Figure $3 b$ shows the RMS errors of the false ocean and the analysis with perturbation factors $\alpha=0.5$ and $\varepsilon_{s}=0.5$ (case 1). In the false ocean, the RMS error in the initial condition decreases in the first $12 \mathrm{~h}$, but then gradually increases again to approximately $0.1 \mathrm{~cm} / \mathrm{s}$ due to the inaccurate wind forcing which evidently plays a fundamental role in affecting the flow. Notice that $0.1 \mathrm{~cm} / \mathrm{s}$ is a domain-averaged RMS error, while the maximum velocity errors can reach $10 \mathrm{~cm} / \mathrm{s}$ within the domain. The analysis RMS error is significantly reduced throughout the 48 h. The normalized error as given by Eq. 2 indicates that the data assimilation reduces the errors up to $90 \%$ after a few hours (Fig. 3c). A remarkable degradation of the error reduction at hour 40 is likely due to the large wind perturbation at that time. Figure $3 \mathrm{~d}$ compares the ensemble spreads for the perturbed and perfect model experiments. The ensemble spread of the perturbed ones is well maintained between $10^{-2}$ and $10^{-3} \mathrm{~cm} / \mathrm{s}$ and is about ten times bigger than for the perfect model.
The results of the perturbed model experiment (case 1 of Table 1) indicate that the success of the EnKF is highly sensitive to the ensemble spread. Figure 4 shows the results of a series of sensitivity experiments with different values of $\alpha$ and $\varepsilon_{s}$ (cases $1-6$ in Table 1 ). In the experiments of $\varepsilon$, $\alpha$ is set to 0.5 and $\varepsilon_{s}=0,0.2$, and 0.5 . The normalized errors calculated as Eq. 2 for $\varepsilon_{s}=0.2$ and 0.5 are similar, while the normalized error for $\varepsilon_{s}=0$ rapidly decreases to zero showing failure of the assimilation. The ensemble spreads of cases 1-3 also confirm that the failure of the EnKF in case 3 is due to the convergence of the ensemble members. In the experiments of $\alpha, \varepsilon_{s}$ is set to 0.2 and $\alpha=0,0.2$, and 0.5 . EnKF succeeds in all three cases, and all ensemble spreads remain the order of $10^{-3} \mathrm{~cm} / \mathrm{s}$. It is noted that the difference between case 6 and the perfect model experiment is the presence of $\varepsilon(0.2)$, indicating that the ineffectiveness of the EnKF in the perfect model can be improved by applying a small random perturbation to each ensemble member.

\section{Summary and discussions}

OSSE experiments are designed to assess the effectiveness of the EnKF data assimilation method in the Selat Pauh of Singapore. Perfect and perturbed model experiments are carried out. Twenty ensemble members are used, based on 
Fig. 4 a Normalized errors and (b) ensemble spreads for $\varepsilon$ experiments (cases 1-3). c Normalized errors and (d) ensemble spreads for $\alpha$ experiments (cases 4-6)
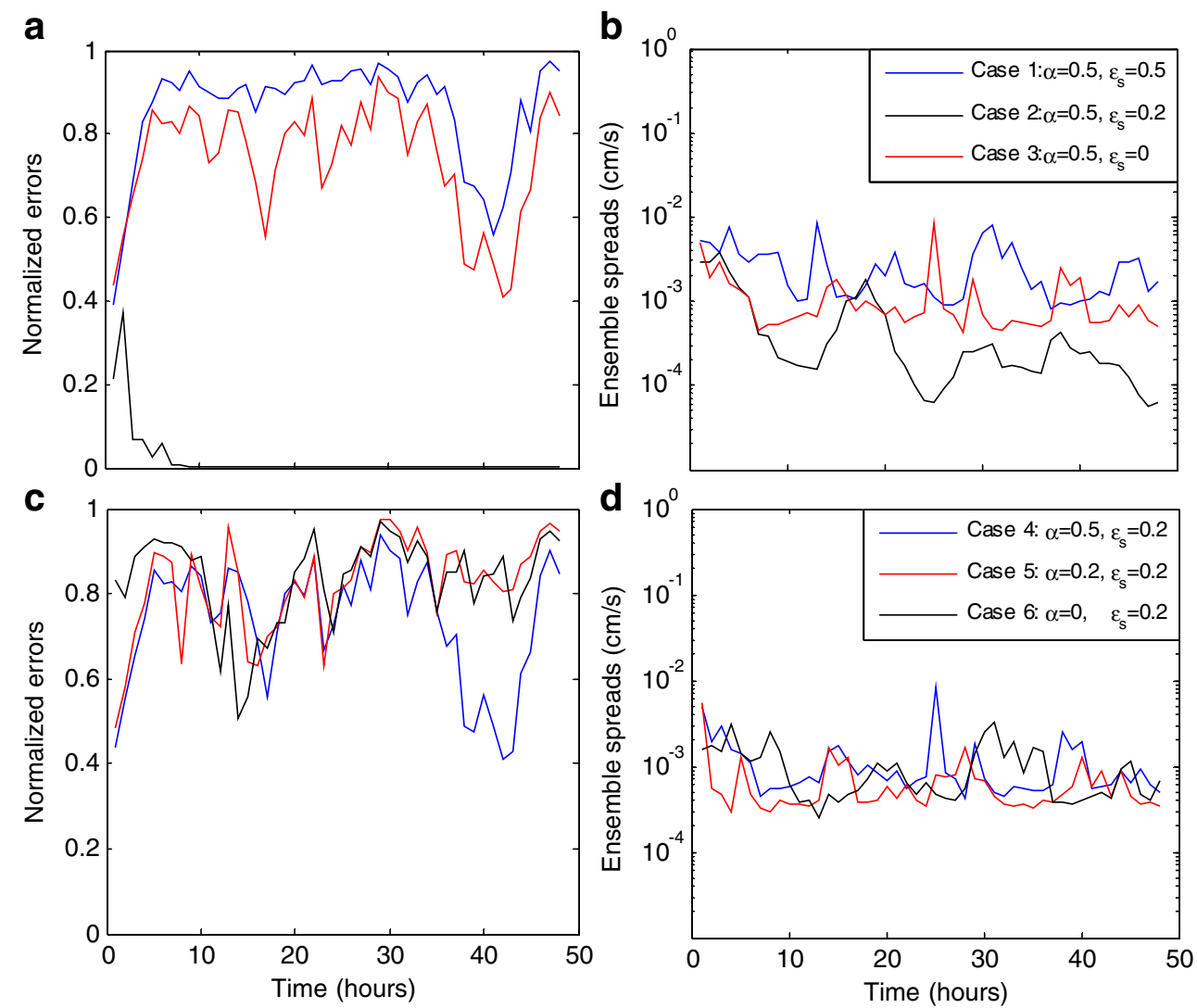

the successful experience in the idealized experiments (Chen et al. 2009). In the perfect model experiments, while the EnKF reduces the initial velocity error in the first few hours, it fails after one tidal cycle due to the rapid convergence of the ensemble members. In the perturbed model experiments, a wrong wind is used as specified in Eq. 1. The EnKF successfully reduces the velocity errors throughout the 48-h assimilation run in the presence of a random perturbation $\varepsilon$ applied independently to each ensemble member. The assimilation-induced error reduction is up to $90 \%$ with respect to the false ocean.

Our model results are consistent with the EnKF theory that the success of the EnKF depends on the diversity of the ensemble members. Since our model only considers the barotropic circulation, the flow evolution is strongly controlled by the boundary tidal forcing. In the perfect model experiments, the tidal forcing is identical to the true ocean, and thus the ensemble members converge rapidly in spite of initial perturbations. In the experiments with the perturbed wind, the tidal forcing is the same. The presence of $\varepsilon$ is essential for the effectiveness of the EnKF as the EnKF succeeds in all the cases including it but fails in the case without. The results also suggest that the success of EnKF is not seriously sensitive to the magnitude of $\varepsilon$, but to its presence, at least for the range of considered values, indicating that in this range, the effect of wind forcing is linear. The degradation of the normalized error observed in Fig. 3c, due to the very large wind perturbation, shows the emergence of nonlinear effects, arguably equivalent to considering much higher values of this perturbation parameter.

Houtekamer and Mitchell (1998) found that the ensemble members tend to converge if the same unperturbed observations are used to update the ensemble analysis. To prevent the convergence of the ensemble members, they proposed to update the analysis with a perturbed set of observations different for each ensemble member. Burgers et al. (1998) and Whitaker and Hamill (2002) also discussed the need of perturbed observations. A recently developed version of the EnKF, the square root filter (SRF; Hoffman et al. 2008), allows maintaining the ensemble spread by directly updating the analysis covariance matrix and by inflating the covariance. The SRF therefore needs no artificial perturbed observations for maintaining the ensemble spread. Our model results showed that for a barotropic regime, even with a set of observations differently perturbed for each ensemble member, the ensemble members still converge rapidly in a few hours, which causes the failure of the EnKF in the perfect and perturbed model experiments (case 3). Differently from Hoffman et al., however, we have added a small random perturbation on wind stress to each ensemble member 
which effectively prevents their convergence and insures the effectiveness of the EnKF. In these experiments, we have focused exclusively on the EnKF. A possible comparison between the EnKF and other possible versions of the filter, such as the SRF, is well beyond the scope of the present investigation.

As a concluding remark, we point out again that the barotropic flow in our simulations is dominated by the tidal boundary forcing. A natural extension would be to reverse the role of tides and wind by perturbing the tidal forcing and leaving the wind unchanged. This extension would require a much greater sensitivity analysis to cover the much broader amplitude/phase perturbation ranges of the prescribed boundary tides. This scenario would however correspond to a rather unrealistic situation as tides, being fully deterministic, are known with a much greater accuracy than the wind field over a small, regional domain affected by land-sea interactions, and this is the reason why we have not addressed this further problem.

Acknowledgments This study was funded by the Office of Naval Research (ONR) and by the Singapore National Research Foundation (NRF) through the Singapore-MIT Alliance for Research and Technology (SMART) and Center for Environmental Sensing and Monitoring (CENSAM).

\section{References}

Anderson JL (2001) An ensemble adjustment filter for data assimilation. Mon Weather Rev 129:2884-2903

Burgers G, van Leeuwen PJ, Evensen G (1998) Analysis scheme in the ensemble Kalman filter. Mon Weather Rev 126:1719-1724

Chen C, Liu H, Beardsley RC (2003) An unstructured, finite-volume, three-dimensional, primitive equation ocean model: application to coastal ocean and estuaries. J Atmos Ocean Technol 20:159-186
Chen C, Beardsley RC, Cowles G (2006a) An unstructured grid, finite-volume coastal ocean model-FVCOM user manual, School for Marine Science and Technology, University of Massachusetts Dartmouth, New Bedford, Second Edition. Technical Report SMAST/UMASSD-06-0602, p 318

Chen C, Beardsley RC, Cowles G (2006b) An unstructured grid, finite-volume coastal ocean model (FVCOM) system. Special issue entitled Advance in Computational Oceanography. Oceanography 19(1):78-89

Chen C, Malanotte-Rizzoli P, Wei J, Beardsley RC, Lai Z, Xue P, Lyu S, Xu Q, Qi J, Cowles G (2009) Comparison and validation of Kalman filters for coastal ocean problems: an experiment with FVCOM. J Geophys Res 114:C05011. doi: $10.1029 / 2007 \mathrm{JC} 004548$

Evensen G (1994) Sequential data assimilation with a nonlinear quasigeostrophic model using Monte Carlo methods to forecast error statistics. J Geophys Res 99(10):143-10 162

Hamill TM, Whitaker JS, Snyder C (2001) Distance-dependent filtering of background error covariance estimates in an ensemble Kalman filter. Mon Weather Rev 129:2776-2790

Gaspari G, Cohn SE (1999) Construction of correlation functions in two and three dimensions. Quart J Roy Meteor Soc 125:723-757

Hoffman RN, Ponte RM, Kostelich EJ, Blumberg A, Szunyogh I (2008) A simulation study using a local ensemble transform Kalman filter for data assimilation in New York Harbor. J Atmos Ocean Technol 25:1638-1656

Houtekamer P, Mitchell HL (1998) Data assimilation using an ensemble Kalman filter technique. Mon Weather Rev 126:796811

Kalnay E (2003) Atmospheric modeling, data assimilation, and predictability. Cambridge University Press, Cambridge, p 341

Tippett MK, Anderson JL, Bishp CH, Hamill T, Whitaker JS (2003) Ensemble square root filters. Mon Weather Rev 131:1485-1490

Tkalich P, Vethamony P, Babu MT, Pokratath R (2009) Seasonal sea level variability and anomalies in the Singapore Strait. Proceedings of International Conference in Ocean Engineering, ICOE

Whitaker JS, Hamill TM (2002) Ensemble data assimilation withou perturbed observations. Mon Weather Rev 130:1913-1924

Zang X, Malanotte-Rizzoli P (2003) A comparison of assimilation results from the ensemble Kalman filter and the reduced-rank extended Kalman filter. Nonlinear Process Geophys 10:6,4776,491 